\title{
AN INTEGRAL EQUATION TECHNIQUE FOR SOLVING MIXED BOUNDARY VALUE PROBLEMS
}

We discuss here the solution of $n(n>1)$ simultaneous Fredholm integral equations of the first kind which cannot be solved directly and present an integral equation method to convert them into $4 n$ Volterra integral equations of the first kind and $4 n$ Fredholm integral equations of the second kind. The $4 n$ Volterra integral equations have simple kernels and can therefore be easily inverted whereas the $4 n$ Fredholm integral equations of the second kind can be solved approximately by iteration. Finally, we illustrate our method by considering an electrostatic potential problem involving two annular coaxial concentric spherical caps in a free space.

1. Introduction. Various methods have been developed to solve mixed boundary value problems in mechanies and mathematical physies (see [1], [2], [8], [13]-[15], [19], [20]). One of the methols was originated by Williams [19] and [20] and was later modified by Jain and Kanwal [8]. In this method the solution of a mixed boundary value problem is expressed in terms of the solution of a Fredholm integral equation of the first kind. Later, by using an interesting technique, the integral equation is converted into a set of Fredholm integral equation of the second kind and Volterra integral equations of the first kind. Using this technique, several mixed boundary value problems were solved successfully (see [4]-[7] and [9]-[11]). All these boundary value problems were associated with only one object. Vaid and Jain [16] presented an integral equation technique to consider $2 n$ part boundary value problems which arise in connection with $n$ coaxial disks or $n$ coaxial concentric spherical caps. They have solved various boundary value problems involving two coaxial disks or two concentric coaxial spherical caps (see [17] and [18]).

The objective of this paper is to connect the integral equation techniques given in [8] and [16]. We propose to develop an integral equation method which is useful in discussing mixed boundary value problems which arise in connection with $n$ coaxial annular disks or $n$ annular coaxial 
concentric spherical caps. Thus it represents a development of Williams work [19].

By following Green's function approach [12], we express the solution of the problem in terms of the solution of $n$ simultaneous Fredholm integral equations of the first kind which cannot be solved directly. Following the techniques of [8] and [16] and splitting the kernels in the given $n$ Fredholm integral equations of the first kind, we readily convert their solutions to the solutions of $4 n$ Fredholm integral equations of the second kind and $4 n$ Volterra integral equations of the first kind. The Volterra integral equations have simple kernels and thus can be easily inverted, and the Fredholm integral equations of the second kind can be solved approximately by standard iterations in terms of small perturbation parameters.

The scheme of this paper is as follows. In Section 2 we present the method of solving the generalized $3 n$ part boundary value problems. In order to illustrate the method we consider the electrostatic potential problem of two coaxial concentric annular spherical caps charged to prescribed potentials in a free space. By using this method we can solve many problems occurring in electrostatics, acoustics as well as in electromagnetic diffraction and hydrodynamies for the cases where they are associated with several annular objects and where the corresponding problem for one object is already solved. In fact, the author has already solved some of these problems and they will be the subject of future communications.

2. Integral equation method. Solutions of many mixed boundary value problems in mechanics and mathematical physics, associated with $n$ coaxial disks, are described by (see [12]) the equation

$$
\begin{array}{r}
\int_{b_{i}}^{a_{i}} K_{i i}(t, \varrho) g_{i}(t) d t+\sum_{\substack{j=1 \\
j \neq i}}^{n} \int_{b_{i}}^{a_{i}} G_{i j}(t, \varrho) g_{j}(t) d t=f_{i}(\varrho), \quad b_{i}<\varrho<a_{i} \\
(i=1,2, \ldots, n),
\end{array}
$$

where $b_{i}$ and $a_{i}$ are the inner and the outer radii, respectively, of the $i$-th disk $(i=1,2, \ldots, n), f_{i}, K_{i i}$ and $G_{i j}$ are known functions in the integral equations (1), and $g_{i}(t)(i=1,2, \ldots, n)$ are the functions to be determined. All the equations in this paper are valid for $i=1,2, \ldots, n$ unless otherwise stated. These $n$ equations cannot easily be solved for $g_{i}(t)$. However, we can convert (1) into $4 n$ Volterra integral equations of the first kind and $4 n$ Fredholm integral equations of the second kind.

Assuming (see [3])

$$
f_{i}(\varrho)=\sum_{j=-\infty}^{\infty} a_{i j} \varrho^{j}, \quad b_{i}<\varrho<a_{i},
$$


we introduce the $4 n$ functions as

$$
\begin{gathered}
f_{i 1}(\varrho)=\sum_{j=0}^{\infty} a_{i j} \varrho^{j}, \quad 0<\varrho<a_{i}, \\
f_{i 2}(\varrho)=\sum_{j=-\infty}^{-1} a_{i j} \varrho^{j}, \\
g_{i 1}(t)+g_{i 2}(t)= \begin{cases}0, & 0<t<b_{i}, \\
g_{i}(t), & b_{i}<t<a_{i}, \\
0, & a_{i}<t<\infty,\end{cases}
\end{gathered}
$$

thus splitting (1) into the following $2 n$ equations (6) and (7):

$$
\begin{aligned}
& \int_{0}^{\infty} K_{i i}(t, \varrho) g_{i 1}(t) d t+\sum_{\substack{j=1 \\
j \neq i}}^{n} \int_{0}^{\infty} G_{i j}(t, \varrho) g_{j 1}(t) d t=f_{i 1}(\varrho), \quad 0<\varrho<\infty, \\
& \int_{0}^{\infty} K_{i i}(t, \varrho) g_{i 2}(t) d t+\sum_{\substack{j=1 \\
j \neq i}}^{n} \int_{0}^{\infty} G_{i j}(t, \varrho) g_{j 2}(t) d t=f_{i 2}(\varrho), \quad 0<\varrho<\infty .
\end{aligned}
$$

This method requires the kernels $K_{i j}$ and $G_{i j}$ to satisfy the following two conditions:

(a) $K_{i i}$ can be split into the form

$$
K_{i i}=L_{i}+G_{i i},
$$

where $G_{i j}$ are in some sense smaller than the dominating part $L_{i}$ of the kernel $K_{i i}$;

(b) the kernels $L_{i}$ are expressible in the following forms:

(9) $\quad L_{i}(t, \varrho)$

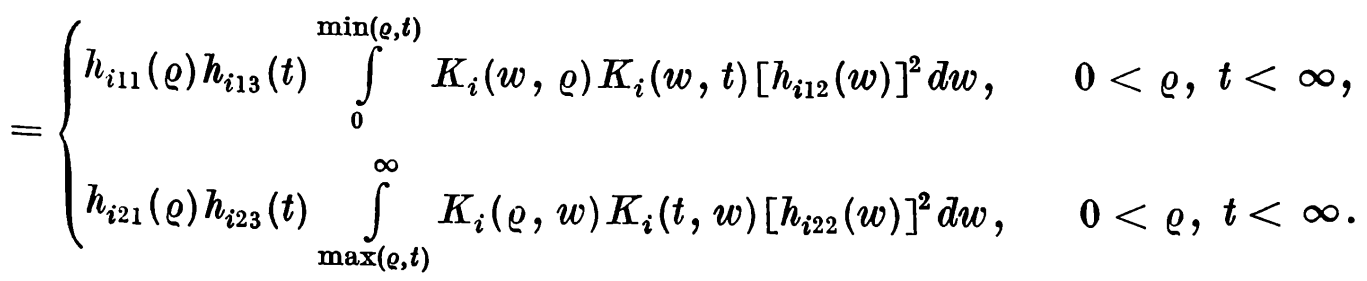

Here $h_{i k j}(k, j=1,2,3)$ and $K_{i}$ are known functions such that the $2 n$ Volterra integral equations

$$
\int_{0}^{\varrho} K_{i}(t, \varrho) g(t) d t=F_{1}(\varrho), \quad 0<\varrho<\infty
$$

and

$$
\int_{\varrho}^{\infty} K_{i}(\varrho, t) g(t) d t=F_{2}(\varrho), \quad 0<\varrho<\infty,
$$

8 - Zastosow. Matem. 16.1 
have explicit unique solutions for $g(t)$ in terms of the arbitrary differentiable functions $F_{1}$ and $F_{2}$. It is obvious that relations (10) and (11) ensure that the kernels $G_{i j}$ can be expressed as

$$
\begin{aligned}
& G_{i j}(t, \varrho) \\
& h_{i 11}(\varrho) h_{i 13}(t) \int_{0}^{e} \int_{\theta}^{t} K_{i}(w, \varrho) K_{i}(v, t) h_{i 12}(w) h_{i 12}(v) L_{i j 1}(v, w) d v d w, \\
& h_{i 21}(\varrho) h_{i 23}(t) \int_{\varrho}^{\infty} \int_{i}^{\infty} K_{i}(\varrho, w) K_{i}(t, v) h_{i 22}(w) h_{i 22}(v) L_{i j 2}(v, w) d v d w .
\end{aligned}
$$

Using (8), (9) and (12) in equations (6) and (7), and interchanging various orders of integrations, we transform these equations into

$$
\begin{gathered}
h_{i 11}(\varrho)\left[\int_{0}^{\infty} K_{i}(w, \varrho)\left\{h_{i 12}(w)\right\}^{2} \int_{w}^{\infty} K_{i}(w, t) g_{i 1}(t) h_{i 13}(t) d t d w+\right. \\
+\sum_{j=1}^{n} \int_{0}^{\varrho} K_{i}(w, \varrho) h_{i 12}(w) \int_{0}^{\infty} L_{i j 1}(v, w) h_{i 12}(v) \int_{v}^{\infty} K_{i}(v, t) \times \\
\left.\times g_{j 1}(t) h_{i 13}(t) d t d v d w\right]=f_{i 1}(\varrho), \quad 0<\varrho<a_{i}, \\
h_{i 21}(\varrho)\left[\int_{\varrho}^{\infty} K_{i}(\varrho, w)\left\{h_{i 22}(w)\right\}^{2} \int_{0}^{w} K_{i}(t, w) g_{i 2}(t) h_{i 23}(t) d t d w+\right. \\
+\sum_{j=1}^{n} \int_{\varrho}^{\infty} K_{i}(\varrho, w) h_{i 22}(w) \int_{0}^{\infty} L_{i j 2}(v, w) h_{i 22}(v) \int_{0}^{v} K_{i}(t, v) \times \\
\left.\times g_{j 2}(t) h_{i 23}(t) d t d v d w\right]=f_{i 2}(\varrho), \quad b_{i}<\varrho<\infty .
\end{gathered}
$$

Finally, we define $6 n$ functions $S_{i k}, T_{i k}$, and $C_{i k}(k=1,2)$ so that

$$
\begin{aligned}
& h_{i 12}(\varrho) \int_{\varrho}^{\infty} K_{i}(\varrho, t) g_{i 1}(t) h_{i 13}(t) d t= \begin{cases}S_{i 1}(\varrho), & 0<\varrho<a_{i}, \\
-T_{i 1}(\varrho), & a_{i}<\varrho<\infty,\end{cases} \\
& h_{i 22}(\varrho) \int_{0}^{\varrho} K_{i}(t, \varrho) g_{i 2}(t) h_{i 23}(t) d t= \begin{cases}-T_{i 2}(\varrho), & 0<\varrho<b_{i}, \\
S_{i 2}(\varrho), & b_{i}<\varrho<\infty,\end{cases} \\
& h_{i 11}(\varrho) \int_{0}^{\varrho} K_{i}(w, \varrho) C_{i 1}(w) h_{i 12}(w) d w=f_{i 1}(\varrho), \quad 0<\varrho<a_{i} \text {, } \\
& h_{i 21}(\varrho) \int_{\varrho}^{\infty} K_{i}(\varrho, w) C_{i 2}(w) h_{i 22}(w) d w=f_{i 2}(\varrho), \quad b_{i}<\varrho<\infty .
\end{aligned}
$$

It is obvious that the expressions for the $2 n$ functions $C_{i k}(\varrho)$ can explicitly be obtained from equations (17) and (18) in view of the assumptions that equations (10) and (11) can be inverted. Using (15)-(18) 
in equations (13) and (14), we obtain

$$
\begin{aligned}
& S_{i 1}(\varrho)+\sum_{j=1}^{n} \int_{0}^{a_{j}} L_{i j 1}(v, \varrho) S_{j 1}(v) d v \\
& \quad=C_{i 1}\left(\varrho^{\prime}+\sum_{j=1}^{n} \int_{a_{j}}^{\infty} L_{i j 1}(v, \varrho) T_{j 1}(v) d v, \quad 0<\varrho<a_{i}\right.
\end{aligned}
$$

$$
\begin{aligned}
S_{i 2}(\varrho)+\sum_{j=1}^{n} \int_{b_{j}}^{\infty} L_{i j 2}(v, \varrho) S_{j 2}(v) d v \\
\quad=C_{i 2}(\varrho)+\sum_{j=1}^{n} \int_{0}^{b_{j}} L_{i j 2}(v, \varrho) T_{j 2}(v) d v, \quad b_{i}<\varrho<\infty .
\end{aligned}
$$

Using equations (5), (15) and (16), we also get

$$
\begin{aligned}
T_{i 1}(\varrho)=h_{i 12}(\varrho) \int_{\varrho}^{\infty} K_{i}(\varrho, t) g_{i 2}(t) h_{i 13}(t) d t, & a_{i}<\varrho<\infty, \\
T_{i 2}(\varrho)=h_{i 22}(\varrho) \int_{0}^{\varrho} K_{i}(t, \varrho) g_{i 1}(t) h_{i 23}(t) d t, & 0<\varrho<b_{i} .
\end{aligned}
$$

By equations (2), (3) and (4), we get $f_{i 1}$ and $f_{i 2}$ which enables us to determine $C_{i 1}$ and $C_{i 2}$ in view of equations (17) and (18). By inverting (15) and (16), we get $g_{i 1}$ and $g_{i 2}$ in terms of $S_{i k}$ and $T_{i k}(k=1,2)$. Substituting these values of $g_{i k}$ into equations (21) and (22) we obtain $2 n$ relations which together with $2 n$ equations (19) and (20) are enough to determine the approximate values of the $4 n$ unknown functions $S_{i k}$ and $T_{i k}(k=1,2)$. Once $S_{i k}$ and $T_{i k}$ are defined, we determine $g_{i k}$ which gives the values of $g_{i}(t)$ in view of relation (5).

Finally, we point out that the above analysis can also be applied to $n$ simultaneous Fredholm integral equations of the first kind,

$$
\int_{\beta_{i}}^{a_{i}} K_{i i}(t, \theta) g_{i}(t) d t+\sum_{\substack{j=1 \\ j \neq i}}^{n} \int_{\beta_{j}}^{\alpha_{j}} G_{i j}(t, \theta) g_{j}(t) d t=f_{i}(\theta), \quad \beta_{i}<\theta<\alpha_{i}
$$

which embodies the solutions of many mixed boundary value problems in mechanics and mathematical physics for $n$ concentric coaxial spherical caps, where we change $\varrho, a_{i}, b_{i}, \infty$ into $\theta, \alpha_{i}, \beta_{i}, \pi$, respectively, $a_{i}, \beta_{i}$ being the bounding angles of the $i$-th annular cap.

So far we have considered the solution of a generalized $3 n$ part boundary value problem. For a $3 n$ part boundary value problem, we have $G_{i i}=0$, and so $L_{i i}=0$, and the rest of the analysis is the same.

We illustrate the method of this section by considering one mixed boundary value problem in electrostatics involving $n$ coaxial concentric annular spherical caps. Later we consider a special case by taking $n=2$. 
3. Electrostatic potential problem for $n$ annular coaxial concentric spherical caps. Let us consider $n$ annular concentric coaxial spherical caps of radii $a_{i}$ with inner and outer bounding angles $\beta_{i}$ and $\alpha_{i}$, respectively. Let the $i$-th annular cap be charged to an axially symmetric potential $f_{i}(\theta)$ in a free space. Let $V(r, \theta, \varphi)$ denote the potential of the system in spherical polar coordinates $(r, \theta, \varphi)$ with $\theta=0$ as the axis of symmetry, origin being at their common centre. Then we have the boundary problem

$$
\begin{gathered}
\nabla^{2} V(r, \theta, \varphi)=0 \quad \text { in } D, \\
V\left(a_{i}, \theta, \varphi\right)=f_{i}(\theta) \quad \text { for all } \beta_{i}<\theta<\alpha_{i},
\end{gathered}
$$

where $V$ and $\partial V / \partial r$ are continuous across the region $r=a_{i}, 0<\theta<\beta_{i}$, $\alpha_{i}<\theta<\pi$, and $D$ being the region exterior to all the caps.

Using Green's function approach [12], we get

$$
V(r, \theta, \varphi)=\sum_{j=1}^{n} a_{j}^{2} \int_{\beta_{j}}^{a_{j}} \int_{0}^{2 \pi}\left[\sin t \frac{\sigma_{j}(t)}{R\left(r, \theta, \varphi ; a_{j}, t, \varphi^{\prime}\right)}\right] d t d \varphi^{\prime},
$$

where $R\left(r, \theta, \varphi ; a_{j}, t, \varphi^{\prime}\right)$ is the distance between the points $(r, \theta, \varphi)$ and $\left(a_{j}, t, \varphi^{\prime}\right)$, and $\sigma_{j}(t)$ are the charge densities.

By the boundary conditions (23), we obtain the following $n$ simultaneous Fredholm integral equations of the first kind:

$$
\begin{aligned}
a_{i}^{2} & \int_{\beta_{i}}^{a_{i}} \int_{0}^{2 \pi}\left[\sin t \frac{\sigma_{i}(t)}{R\left(a_{i}, \theta, \varphi ; a_{i}, t, \dot{\varphi}^{\prime}\right)}\right] d \varphi^{\prime} d t+ \\
+ & \sum_{\substack{j=1 \\
j \neq i}}^{n} a_{j}^{2} \int_{\beta_{j}}^{a_{j}} \int_{0}^{2 \pi}\left[\sin t \frac{\sigma_{j}(t)}{R\left(a_{i}, \theta, \varphi ; a_{j}, t, \varphi^{\prime}\right)}\right] d \varphi^{\prime} d t=f_{i}(\theta), \quad \alpha_{i}<\theta<\beta_{i} .
\end{aligned}
$$

Comparing these equations with the notation in the previous section, and using some well-known results [10], we have

$$
\begin{gathered}
K_{i i}(t, \theta)=L_{i}(t, \theta)=a_{i} \int_{0}^{2 \pi} \frac{d \varphi^{\prime}}{R\left(a_{i}, \theta, \varphi ; a_{i}, t, \varphi^{\prime}\right)}, \quad G_{i i}=0, \\
G_{i j}(t, \theta)=a_{j} \int_{0}^{2 \pi} \frac{d \varphi^{\prime}}{R\left(a_{i}, \theta, \varphi ; a_{j}, t, \varphi^{\prime}\right)}, \\
a_{i} \sin t \sigma_{i k}(t)=g_{i k}(t) \quad(k=1,2), \\
h_{i 11}=h_{i 21}=2, \quad h_{i 13}=h_{i 12}=h_{i 23}=h_{i 22}=1, \quad K_{i}(w, \theta)=(\cos w-\cos \theta)^{-1 / 2}
\end{gathered}
$$


with

$$
\begin{aligned}
& L_{i j 1}(v, w)=\frac{2 a_{j}}{\pi} \sum_{n=0}^{\infty} \frac{a_{\leqslant}^{n}}{a_{\geqslant}^{n+1}}\left[\cos \left(n+\frac{1}{2}\right) w\right]\left[\cos \left(n+\frac{1}{2}\right) v\right], \\
& L_{i j 2}(v, w)=\frac{2 a_{j}}{\pi} \sum_{n=0}^{\infty} \frac{a_{\leqslant}^{n}}{a_{\geqslant}^{n+1}}\left[\sin \left(n+\frac{1}{2}\right) w\right]\left[\sin \left(n+\frac{1}{2}\right) v\right],
\end{aligned}
$$

and

$$
a_{\leqslant}=\min \left(a_{i}, a_{j}\right), \quad a_{\geqslant}=\max \left(a_{i}, a_{j}\right) .
$$

The kernel $K_{i}(w, \theta)$ is elementary and the equations corresponding to (15)-(18) can easily be inverted [12].

We now consider the particular case where $n=2$. Suppose that the two annular caps of radii $a_{1}$ and $a_{2}$ are kept at constant potentials $V_{1}$ and $V_{2}$, respectively. We further assume that $a_{1} / a_{2}=\lambda$ and that $\beta_{1}, \beta_{2}, \lambda \ll 1$.

In this case, we have $f_{i 1}(\theta)=V_{i}, f_{i 2}(\theta)=0(i=1,2)$ for the rest of the analysis, and

$$
C_{i 1}(\theta)=-\frac{1}{\sqrt{2} \pi} V_{i} \cos \frac{\theta}{2}, 0<\theta<a_{i}, \quad \text { and } \quad C_{i 2}(\theta)=0, \beta_{i}<\theta<\pi
$$

To solve equations (19)-(22), we assume that $S_{i 1}=X_{i 1}+W_{i 1}$, where, for $i, j=1,2, i \neq j$,

$$
\begin{gathered}
X_{i 1}(\theta)+\int_{0}^{a_{j}} L_{i j 1}(\theta, \varphi) X_{j 1}(\varphi) d \varphi=C_{i 1}(\theta), \quad 0<\theta<\alpha_{i} \\
W_{i 1}(\theta)+\int_{0}^{a_{j}} L_{i j 1}(\theta, \varphi) W_{j 1}(\varphi) d \varphi=\int_{a_{j}}^{\pi} L_{i j 1}(\theta, \varphi) T_{j 1}(\varphi) d \varphi, \quad 0<\theta<\alpha_{i} .
\end{gathered}
$$

Equations (24) and (25) have the solutions (see [16])

$$
\begin{aligned}
X_{i 1}(\theta)= & \frac{1}{\sqrt{2} \pi}\left[e_{i 1} \cos \frac{\theta}{2}+e_{i 3} \cos \frac{3 \theta}{2}+\right. \\
& \left.+e_{i 5} \cos \frac{5 \theta}{2}+e_{i 7} \cos \frac{7 \theta}{2}+O\left(\lambda^{4}\right)\right]
\end{aligned}
$$

where

$$
\begin{aligned}
& e_{11}=K_{1}\left[1+\frac{\lambda}{\pi^{2}} W_{0}\left(\alpha_{1}\right) W_{0}\left(\alpha_{2}\right)+\frac{\lambda^{2}}{\pi^{4}}\left\{W_{0}\left(\alpha_{1}\right) W_{0}\left(\alpha_{2}\right)\right\}^{2}+\frac{\lambda^{2}}{\pi^{2}} W_{1}\left(a_{1}\right) W_{1}\left(\alpha_{2}\right)+\right. \\
& \left.+\frac{\hat{\lambda}^{3}}{\pi^{2}}\left\{W_{2}\left(a_{1}\right) W_{2}\left(\alpha_{2}\right)+\frac{3}{\pi^{2}} W_{0}\left(\alpha_{1}\right) W_{1}\left(\alpha_{1}\right) W_{0}\left(a_{2}\right) W_{1}\left(a_{2}\right)+\frac{1}{\pi^{4}} W_{0}^{3}\left(a_{1}\right) W_{0}^{3}\left(\alpha_{2}\right)\right\}\right]-
\end{aligned}
$$




$$
\begin{aligned}
& -V_{2} \frac{\lambda^{2}}{\pi^{3}}\left[W_{1}\left(\alpha_{2}\right) W_{0}\left(\alpha_{2}\right) W_{1}\left(\alpha_{1}\right)+\lambda W_{1}^{2}\left(\alpha_{2}\right) A\left(\alpha_{1}\right)+\lambda W_{0}\left(\alpha_{2}\right) W_{2}\left(\alpha_{2}\right) W_{2}\left(\alpha_{1}\right)+\right. \\
& \left.+\frac{\lambda}{\pi^{2}} W_{0}\left(a_{1}\right) W_{1}\left(\alpha_{1}\right) W_{1}\left(\alpha_{2}\right) W_{0}^{2}\left(a_{2}\right)\right] \\
& e_{13}=K_{1} \frac{\lambda^{2}}{\pi^{2}}\left[W_{1}\left(\alpha_{2}\right) W_{0}\left(\alpha_{1}\right)+\lambda A\left(\alpha_{2}\right) W_{1}\left(\alpha_{1}\right)+\frac{\lambda}{\pi^{2}} W_{0}^{2}\left(\alpha_{1}\right) W_{0}\left(\alpha_{2}\right) W_{1}\left(\alpha_{2}\right)\right]- \\
& -V_{2} \frac{\lambda}{\pi} W_{1}\left(a_{2}\right)\left[1+\frac{\lambda^{2}}{\pi^{2}} W_{1}\left(a_{2}\right) W_{1}\left(a_{1}\right)\right] \\
& e_{15}=\frac{\lambda^{2}}{\pi} W_{2}\left(\alpha_{2}\right)\left[-V_{2}+\frac{\lambda}{\pi} K_{1} W_{0}\left(a_{1}\right)\right] \\
& e_{17}=-V_{2} \frac{\lambda^{3}}{\pi} W_{3}\left(a_{2}\right) \\
& e_{21}=K_{2}\left[1+\frac{\lambda}{\pi^{2}} W_{0}\left(a_{1}\right) W_{0}\left(a_{2}\right)+\frac{\lambda^{2}}{\pi^{2}} W_{1}\left(a_{1}\right) W_{1}\left(a_{2}\right)+\frac{\lambda^{3}}{\pi^{2}} W_{2}\left(a_{1}\right) W_{2}\left(a_{2}\right)+\right. \\
& +\frac{\lambda^{2}}{\pi^{4}} W_{0}^{2}\left(a_{1}\right) W_{0}^{2}\left(a_{2}\right)+\frac{3 \lambda^{3}}{\pi^{4}} W_{1}\left(a_{1}\right) W_{0}\left(\alpha_{1}\right) W_{0}\left(a_{2}\right) W_{1}\left(\alpha_{2}\right)+ \\
& \left.+\frac{\lambda^{3}}{\pi^{6}} W_{0}^{3}\left(\alpha_{1}\right) W_{0}^{3}\left(\alpha_{2}\right)\right]-\nabla_{1} \frac{\lambda^{3}}{\pi^{3}} W_{1}\left(\alpha_{1}\right) W_{0}\left(\alpha_{1}\right) W_{1}\left(\alpha_{2}\right), \\
& e_{23}=K_{2} \frac{\lambda^{2}}{\pi^{2}}\left[W_{1}\left(a_{1}\right) W_{0}\left(a_{2}\right)+\lambda A\left(a_{1}\right) W_{1}\left(a_{2}\right)+\frac{\lambda}{\pi^{2}} W_{0}\left(a_{1}\right) W_{0}^{2}\left(a_{2}\right) W_{1}\left(a_{1}\right)\right]- \\
& -V_{1} \frac{\lambda^{2}}{\pi} W_{1}\left(a_{1}\right) \\
& e_{25}=-V_{1} \frac{\lambda^{3}}{\pi} W_{2}\left(a_{1}\right)+\lambda^{3} K_{2} W_{2}\left(a_{1}\right) W_{0}\left(a_{2}\right), \\
& e_{27}=0
\end{aligned}
$$

with

$$
\begin{gathered}
W_{n}(\alpha)=\left\{\begin{array}{cc}
\frac{\sin n a}{n}+\frac{\sin (n+1) \alpha}{n+1}, & n \neq 0, \\
\alpha+\sin \alpha, & n=0,
\end{array}\right. \\
K_{1}=V_{1}-V_{2} \frac{1}{\pi} W_{0}\left(a_{2}\right), \quad K_{2}=V_{2}-\nabla_{1} \frac{\lambda}{\pi} W_{0}\left(a_{1}\right)
\end{gathered}
$$

and

$$
A(a)=\alpha+\frac{1}{3} \sin 3 \alpha
$$


The other functions are obtained in the following order: $X_{i 1}, l_{i 1}$, $T_{i 2}, S_{i 2}, l_{i 2}, T_{i 1}, W_{i 1}, S_{i 1}$. The results are

$$
\begin{aligned}
l_{i 1} & =T_{i 2}=e_{i 1} \frac{1}{2 \sqrt{2} \pi^{2}}\left[\alpha_{i}+2 \cot \frac{\alpha_{i}}{2}\right] \theta+O\left(\beta_{i}^{2}\right), \quad 0<\theta<\beta_{i}, \\
S_{12} & =e_{21} \frac{1}{6 \sqrt{2} \pi^{3}}\left[\alpha_{2}+2 \cot \frac{\alpha_{2}}{2}\right] \beta_{2}^{3} \sin \left(\frac{\theta}{2}\right)+O\left(\beta_{2}^{4}\right), \quad \beta_{1}<\theta<\pi, \\
S_{22} & =O\left(\beta_{2}^{4}\right), \quad \beta_{2}<\theta<\pi, \\
l_{12} & =e_{21} \frac{1}{6 \sqrt{2} \pi^{3}}\left[\alpha_{2}+2 \cot \frac{\alpha_{2}}{2}\right] \beta_{2}^{3} \cos \left(\frac{\theta}{2}\right)+O\left(\beta_{1}^{4}\right), \quad \alpha_{1}<\theta<\pi, \\
l_{22} & =O\left(\beta_{1}^{4}\right), \quad \alpha_{2}<\theta<\pi, \\
T_{i 1} & =l_{i 2}+e_{i 1} \frac{1}{12 \sqrt{2} \pi^{3}} \cot \left(\frac{\theta}{2}\right) \operatorname{cosec}\left(\frac{\theta}{2}\right) \beta_{i}^{3}\left[\alpha_{i}+2 \cot \frac{\alpha_{i}}{2}\right], \quad \alpha_{i}<\theta<\pi, \\
S_{11} & =X_{11}+e_{21} \beta_{2}^{3} \frac{1}{6 \sqrt{2} \pi^{4}}\left[\alpha_{2}+2 \cot \frac{\alpha_{2}}{2}\right]\left[\alpha_{2}+2 \cot \left(\frac{\alpha_{2}}{2}\right)-\pi\right] \cos \frac{\theta}{2}, \\
S_{21} & =X_{21}+O\left(\beta_{1}^{4}\right) .
\end{aligned}
$$

Finally, we calculate the total charge $C_{i}$ on each of the annular caps. Indeed, following the same method as given in appendix $B$ of [10], we get

$$
\begin{aligned}
C_{i}= & 2 \pi a_{i}^{2} \int_{\beta_{i}}^{a_{i}} \sin \theta \sigma_{i}(\theta) d \theta \\
= & 2 \sqrt{2}\left[\int_{0}^{a_{i}} \cos \left(\frac{w}{2}\right) S_{i 1}(w) d w-\int_{a_{i}}^{\pi} \cos \left(\frac{w}{2}\right) T_{i 1}(w) d w-\right. \\
& \left.-\int_{0}^{\beta_{i}} \sin \left(\frac{w}{2}\right) T_{i 2}(w) d w+\int_{\beta_{i}}^{\pi} \sin \left(\frac{w}{2}\right) S_{i 2}(w) d w\right]
\end{aligned}
$$

These integrals can easily be evaluated.

The capacity of the condenser can be obtained from the previous results by taking $V_{1}=V_{2}=1$ and by adding the two total charges thus obtained. Here we see that, if we take $\beta_{1}, \beta_{2} \rightarrow 0$, we get the known results.

By giving special values to the bounding angles of the annular spherical caps we can consider several cases of interest most of which appear to be new.

Following this technique we can present the corresponding generalized $3 n$ part boundary value problems when the caps are surrounded either by a grounded cylinder with axis of symmetry as $\theta=0$ or by two grounded parallel planes. 
The author is grateful to Prof. S. M. Farouq Ali and Prof. R. P. Kanwal for their encouragements. He is also very grateful to Prof. D. L. Jain for advice and for providing him preprints of his research.

\section{References}

[1] W. D. Collins, On the solution of some axisymmetric boundary value problems by means of integral equations, Proc. Edinburgh Math. Soc. 13 (1963), p. 235-246.

[2] J. C. Cooke, Some further triple integral equation solutions, ibidem 13 (1963), p. 303-316.

[3] V. S. Gubenko and V. I. Mossakovskii, Pressure on an axially symmetric circular die on an elastic half space, J. Appl. Math. Mech. 24 (1960), p. 477-486.

[4] D. L. Jain and R. P. Kanwal, Torsional oscillations of an elastic half space due to an annular disk and related problems, Internat. J. Engg. Sci. 8 (1970), p. 687-698.

[5] - Acoustic diffraction by a rigid annular disk, J. Engg. Math. 4 (1970), p. $219-228$.

[6] - Electromagnetic diffraction by a thin conducting annular disk, J. Math. Phys. 12 (1971), p. 723-736.

[7] - Williams' integral equation technique with application to mathematical physics, Indian J. Mech. Math., Special issue II (1971).

[8] - An integral equation method for solving mixed boundary value problems, SIAM J. Appl. Math. 20 (1971), p. 642-658.

[9] - Acoustic diffraction by a perfectly soft annular spherical cap, Internat. J. Engg. Sci. 10 (1972), p. 193-211.

[10] - Three part boundary value problems in potential and generalised axially symmetric potential theories, J. d'Analyse Math. 25 (1972), p. 107-158.

[11] - Acoustic diffraction by a rigid annular spherical cap, J. Appl. Mech. 39 (1972), p. 139-147.

[12] R. P. Kanwal, Limear integral equations, Acad. Press, New York 1971.

[13] I. N. Sneddon, Mixed boundary value problems in potential theory, J. Wilcy, New York 1966.

[14] D. P. Thomas, Acoustic diffraction by an annular disk, Internat. J. Engg. Sci. 3 (1965), p. 405-416.

[15] - A note on the torsional oscillations of an elastic half space, ibidem 6 (1968), p. $565-570$.

[16] B. K. Vaid and D. L. Jain, An integral equation technique, SIAM J. Appl. Math. 26 (1974), p. 455-468.

[17] - Some electrostatic and hydrodynamic problems for two spherical caps, ibidem 30 (1976), p. $452-462$.

[18] - Acoustic diffraction by two coaxial soft and rigid circular disks (to appear).

[19] W. E. Williams, The reduction of boundary value problems to Fredholm integral equations of the second kind, ZAMP 13 (1962), p. 133-152.

[20] - Integral equation formulation of some three part boundary value problems, Proc. Edinburgh Math. Soc. 13 (1963), p. 317-323.

DEPT. OF PETROLEUM AND NATURAL GAS ENGG.

PENNSYLVANIA STATE UNIVERSITY

UNIVERSITY PARK, PA. 16802, U.S.A. 
M. L. PAS H A (University Park, Pa.)

\title{
ROZWIAZYWANIE MIESZANYCH ZAGADNIEN BRZEGOWYCH PRZY UŹYCIU RÓ WNAN CAEKO WYCH
}

\author{
STRESZCZENIE
}

W pracy omawia się rozwiązywanie $n(n>1)$ równań całkowych Fredholma pierwszego rodzaju, nie dających się rozwiązać bezpośrednio, i przedstawia się metodę sprowadzenia ich do $4 n$ równań całkowych Volterry pierwszego rodzaju i $4 n$ równań całkowych Fredholma drugiego rodzaju. Równania całkowe Volterry mają jądra proste i moga być łatwo odwrócone, natomiast równania całkowe Fredholma drugiego rodzaju można rozwiązać w sposób przybliżony poprzez iteracje. Proponowaną metodę ilustruje się na przykładzie zagadnienia elektrostatycznego potenojału dla dwu pierścieniowatych współosiowych koncentrycznych czasz sferycznych w przestrzeni swobodnej. 